CERN-PH-TH/2012-227

September 2012

\title{
Jet Fragmentation Function Moments in Heavy Ion Collisions
}

\author{
Matteo Cacciari, ${ }^{1,2}$ Paloma Quiroga-Arias, ${ }^{1}$ Gavin P. Salam ${ }^{3,4,1}$ and Gregory Soyez ${ }^{5}$ \\ ${ }^{1}$ LPTHE, UPMC Univ. Paris 6 and CNRS UMR 7589, Paris, France \\ ${ }^{2}$ Université Paris Diderot, Paris, France \\ ${ }^{3}$ CERN, Department of Physics, Theory Unit, CH-1211 Geneva 23, Switzerland \\ ${ }^{4}$ Department of Physics, Princeton University, Princeton, NJ 08544, USA \\ ${ }^{5}$ Institut de Physique Théorique, CEA Saclay, CNRS URA 2306, F-91191 Gif-sur-Yvette, France
}

\begin{abstract}
The nature of a jet's fragmentation in heavy-ion collisions has the potential to cast light on the mechanism of jet quenching. However the presence of the huge underlying event complicates the reconstruction of the jet fragmentation function as a function of the momentum fraction $z$ of hadrons in the jet. Here we propose the use of moments of the fragmentation function. These quantities appear to be as sensitive to quenching modifications as the fragmentation function directly in $z$. We show that they are amenable to background subtraction using the same jet-area based techniques proposed in the past for jet $p_{t}$ 's. Furthermore, complications due to correlations between background-fluctuation contributions to the jet's $p_{t}$ and to its particle content are easily corrected for.
\end{abstract}




\section{Contents}

1 Introduction

2 Representations of the fragmentation function $\quad 4$

3 Simulation and reconstruction tools

4 Impact of HI background and its subtraction 6

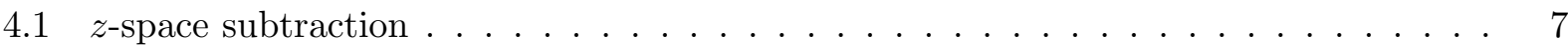

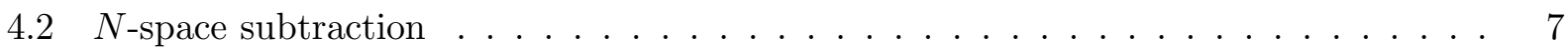

5 Improved background-subtracted fragmentation function 9

6 Conclusions $\quad 13$ 


\section{Introduction}

In heavy-ion physics one is interested in studying the hot and dense medium that is formed by the high energy collisions of heavy nuclei. One way of accessing such properties is to look at how the characteristics of a number of 'hard probes' are modified by their passage through this medium. While early examples of hard probes consisted of quarkonia or single high transversemomentum $\left(p_{t}\right)$ hadrons, the performance and the acceptance of the Large Hadron Collider (LHC) detectors (ALICE, ATLAS and CMS) allow for the in-depth study of fully-fledged jets. A number of experimental results on jets in heavy ion collisions have already been published following the first two heavy ion runs in 2010 and 2011. Of particular interest was the observation by ATLAS [1] and CMS [2, 3] of a sizeable asymmetry in the transverse momentum of dijet pairs, which was interpreted (modulo some caveats [4] concerning a possible partial spoofing role of background fluctuations) as evidence of some amount of quenching, i.e. of a reduction of a jet's momentum due to its interaction with the medium. A better understanding, also at the quantitative level, of this phenomenon would naturally allow one to better constrain the characteristics of the medium itself.

After studying an inclusive observable like the quenching of a full jet, the next logical step is to observe the momentum distribution of its constituents, i.e. its fragmentation function (FF). One would like to ascertain if and how the FF is modified, due to the interactions with the medium, with respect to the $\mathrm{FF}$ of jets produced in proton-proton collisions and fragmenting instead in the vacuum.

Measurements of jet fragmentation functions have been performed since the first observations of hadronic jets. LEP [5, 6, 7] and Tevatron [8, 9] experiments studied them in detail in $e^{+} e^{-}$and $p \bar{p}$ collisions respectively and compared them to accurate calculations in perturbative QCD, finding very good agreement. LHC experiments [10, 11] more recently measured them in $p p$ collisions.

In a heavy ion context, theoretical discussion as to how the fragmentation function could be expected to be modified by the medium were put forward, for instance, in [12, 13, 14, 15, 16, 17, 18, and the question can be examined also with the help of Monte Carlo programs that simulate jet fragmentation in a medium [19, 20, 21, 22, 23. The study of jet FFs in a heavy-ion context is however complicated by the presence of a large and predominantly soft background due to the underlying event produced by the nucleons not directly participating in the hard interaction. The background acts in essentially two ways: it adds many soft particles to the jet - an order of magnitude more than are normally present in a jet - severely contaminating the fragmentation function at low hadron momentum fractions $z$; and it can bias the measured $p_{t}$ of the jet, even after subtracting the expected average $p_{t}$ shift, skewing the value one reconstructs for a given hadron's momentum fraction.

Experimentally, there have so far been measurements of jet FFs by three experiments, in AuAu collisions at RHIC by the STAR collaboration [24, 25], and more recently in $\mathrm{PbPb}$ collisions at the LHC by the CMS [11, 26] and ATLAS [27, 28] collaborations. In each case, the measurements were limited to hadron $p_{t}$ 's above $1-4 \mathrm{GeV}$, where soft contamination is less important. Different approaches were used to address the biases in the determination of $z$, for example unfolding this effect in the case of ATLAS, or folding the effect into the $p p$ reference FF in the case of CMS, the only experiment which goes down to $1 \mathrm{GeV}$. The largest changes of heavy-ion FFs relative to reference $p p$ FFs appear to be in the region of small $z$, where uncertainties associated with the background subtraction grow. It would clearly be of interest to probe the fragmentation functions at lower $z$ and with higher accuracy, especially as other measurements also indicate that soft particles appear to play an important role in quenching [2].

The purpose of this article is to suggest the use of a new observable that may help provide 

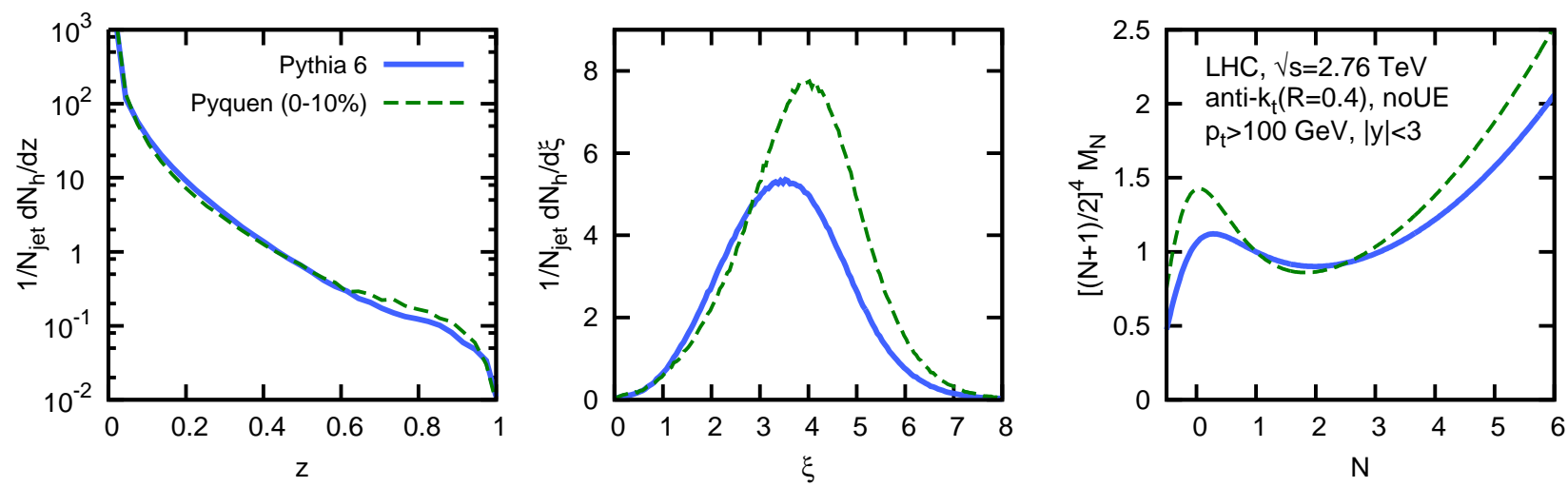

Figure 1: Jet fragmentation functions (versus $z$ and $\xi$ ) and moments (versus $N$ ) in proton-proton collisions at the $\operatorname{LHC}\left(\sqrt{s_{N N}}=2.76 \mathrm{TeV}\right)$, obtained without quenching, from Pythia 6 , and with quenching, from Pyquen.

an alternative way of understanding fragmentation patterns in heavy-ion jets: momentum-fraction weighted moments of the fragmentation functions.

Moments are often used in theoretical calculations, and have been helpful in the past in diagnosing and resolving experiment-theory discrepancies [29], but to our knowledge have not been examined so far in heavy-ion collisions. Among their benefits, one can note that they are helpful in directly relating jet spectra with hadron spectra and also that they are sensible objects on a jet-by-jet basis (whereas event-by-event, fragmentation functions are simply sums of individual delta-functions, one for each hadron in the jet). The latter point makes it feasible to measure correlations within an event between any given moment of the background fragmentation spectrum and fluctuations in the background's $p_{t}$. As we shall see, it seems that this can be of significant benefit in extracting the soft part of the jet's fragmentation and also in reducing systematics in the extraction of the hard part.

\section{Representations of the fragmentation function}

A jet fragmentation function can be defined as the distribution $d N_{h} / d z$ of the momentum fraction

$$
z=\frac{p_{t, h}}{p_{t}}
$$

of hadrons in the jet, where $p_{t, h}$ is the transverse momentum of the hadron and $p_{t}$ is the transverse momentum of the jet 1

Figure 1 shows, in its left-most plot, the typical shape of the $z$ distribution (normalised in this case to the total number of jets $N_{\text {jet }}$ used in the analysis), given for anti- $k_{t}(R=0.4)$ jets 30 ] with $p_{t}>100 \mathrm{GeV}$ in $p p$ collisions at a centre-of-mass energy of $2.76 \mathrm{TeV}$. The middle plot shows identical information, represented as a differential distribution in $\xi=\ln 1 / z$. The $z$ representation helps visualise the hard region of the FF, while $\xi$ devotes more space to the soft part. Two curves are shown: one of them, solid blue, labelled "Pythia 6", is a $p p$ reference curve obtained from Pythia 6.425 [31] with its virtuality-ordered shower. The other, dashed green, was obtained with the Pyquen program [19, 32, which modifies Pythia showering so as to simulate quenching. We

\footnotetext{
${ }^{1}$ Various other definitions are also in use, for example replacing transverse momenta with 3-momenta and taking the projection of the hadron momenta along the axis of the jet.
} 
have used it with settings corresponding to $0-10 \%$ centrality. Its effect on the $\mathrm{FF}$ is of the same order of magnitude as the effects seen experimentally [26, 28] and it therefore provides a useful reference when establishing whether FFs are being reconstructed with sufficient accuracy by some given procedure.

The right-most plot of Fig. 1 1 shows moments of the fragmentation functions. The $N^{\text {th }}$ moment, $M_{N}$, of the fragmentation function is given by the integral

$$
M_{N}=\frac{1}{N_{\text {jet }}} \int_{0}^{1} z^{N} \frac{d N_{h}}{d z} d z=\frac{1}{N_{\text {jet }}} \int_{0}^{\infty} e^{-N \xi} \frac{d N_{h}}{d \xi} d \xi
$$

In practice, the moments for a single jet can be calculated as

$$
M_{N}^{\mathrm{jet}}=\frac{\sum_{i \in \mathrm{jet}} p_{t, i}^{N}}{p_{t}^{N}},
$$

where the sum runs over all the jet's constituents. The results can then be averaged over many jets, so that $M_{N}=\left\langle M_{N}^{\mathrm{jet}}\right\rangle_{\text {jets }}$. Obviously, $M_{0}$ represents the average particle multiplicity in a jet, and $M_{1}$ is equal to one by virtue of momentum conservation if one measures all hadrons, as we assume here (taking $\pi_{0}$ 's to be stable) 2 If instead only charged tracks are used in the numerator, then it is clear that $M_{1}$ will be significantly below 1 . There is another value of $N$ that is of special interest: given a jet spectrum $d \sigma_{\text {jet }} / d p_{t}$ that falls as $p_{t}^{-n}$, the ratio of the inclusive hadron spectrum and inclusive jet spectrum is given by $M_{n-1}$. Thus, $M_{n-1}^{\mathrm{AA}} / M_{n-1}^{\mathrm{pp}}$ corresponds to the ratio of (charged-)hadron and jet $R_{\mathrm{AA}}$ values (in the approximation that $n$ is exactly independent of $p_{t}$ ),

$$
\frac{M_{n-1}^{\mathrm{AA}}}{M_{n-1}^{\mathrm{pp}}}=\frac{R_{\mathrm{AA}}^{\mathrm{h}}}{R_{\mathrm{AA}}^{\mathrm{jet}}} .
$$

For $p_{t}$ in the range $100-200 \mathrm{GeV}$, at $\sqrt{s}_{N N}=2.76 \mathrm{TeV}$, the relevant $n$ value has some dependence on $p_{t}$ and is in the range $n=6-7$, corresponding to $N=5-6$.

In representing the moments in figure 1(right), we include a factor $((N+1) / 2)^{4}$, which allows a broad range of $N$ values to be shown on a linear vertical scale. The same features visible in the plots versus $z$ and $\xi$ are visible versus $N$ too, for example that Pyquen leads to higher multiplicities than Pythia at small $N$ (corresponding to $z<0.05$ or $\xi>3$ ) and large $N(z>0.5, \xi<0.7$ ), and a slightly reduced multiplicity at intermediate $N(z \sim 0.2, \xi \sim 1.5)$.

To help understand the quantitative relationship between $N$ and $\xi$, one may examine figure 2, a colour-map that shows as a function of $N$ the contribution to the $M_{N}$ moment from each $\xi$ value. It shows clearly how large $\xi$ values dominate for low $N$ (and vice-versa). This $\xi, N$ relationship depends to some extent on the shape of the fragmentation function and it is given for the same Pythia 6 fragmentation function that was used in Fig. 1.

\footnotetext{
${ }^{2}$ The relation $M_{1}=1$ is exact only if one defines the transverse momentum of a jet as the scalar sum of the transverse momenta of the constituents, so that the moments are given by

$$
M_{N}^{\mathrm{jet}}=\frac{\sum_{i} p_{t, i}^{N}}{\left(\sum_{i} p_{t, i}\right)^{N}}
$$

This is the choice that has been made in this paper. Using the transverse component of the jet momentum rather than the scalar sum leads to small violations (of the order of a fraction of one percent, for the jet radius $R=0.4$ used in this work) of the $M_{1}=1$ relation. If the fragmentation moments are measured with only charged hadrons, we will still assume that the denominator of Eq. (3) is determined with all particles in the jet or, equivalently, with all calorimeter towers.
} 


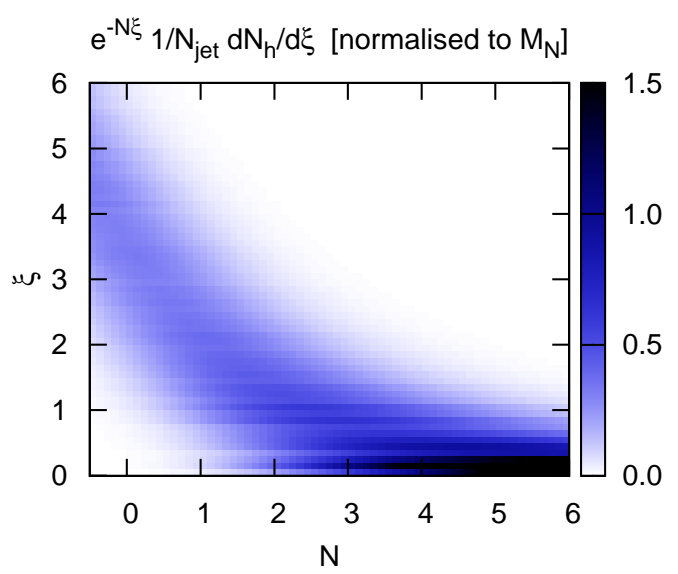

Figure 2: Representation of the $\xi$ values that contribute dominantly to the $M_{N}$ integral for a given $N$, shown as a function of $N$. Shown for the Pythia 6 results and cuts of Fig. 1 .

\section{Simulation and reconstruction tools}

Before proceeding with the heavy-ion analysis, it is useful to detail the simulation tools that we use.

What we call 'hard' jets in QCD are simulated in proton-proton $(p p)$ collisions using Pythia 6.425 [31] in dijet mode with the DW tune [33] ${ }^{3}$ We have not included any $p p$ underlying event (UE), its effect being minimal in this context anyway. Jets including quenching effects have been generated using Pyquen [19, 32], v1.5.1, as included in Hydjet [34, 35]. We consider all hadrons, not just charged tracks, and take $\pi_{0}$ 's to be stable, so that we are still considering genuine hadron distributions.

The heavy-ion background (which we will also call heavy-ion underlying event) is simulated using Hydjet v1.6 for 0-10\% centrality and, where needed, it is superimposed to the hard event generated by Pythia 4 The jets observed in this combined event will be denoted as 'full' jets.

All events are generated for either $p p$ or lead-lead $(\mathrm{PbPb})$ collisions at the LHC, with a centreof-mass energy of $2.76 \mathrm{TeV}$ per nucleon-nucleon collision.

Jets are reconstructed using the anti- $k_{t}$ algorithm [30] with $R=0.4$, as implemented in FastJet [36, 37]. In estimating the jet's $p_{t}$, the HI background is subtracted using the median/area based techniques introduced in [38, 39, 40, which we implement with $k_{t}$ jets [41] $(R=0.4)$ to estimate $\rho$, the background transverse momentum per unit area. At most the two hardest jets passing a hard cut on the subtracted transverse momentum (see below) are subsequently used for the fragmentation function analysis. The cuts that we shall use are $100 \mathrm{GeV}$ and $200 \mathrm{GeV}$.

\section{Impact of HI background and its subtraction}

As explained in the Introduction, the addition of the heavy-ion background has the potential to modify a measured fragmentation function in two ways. Firstly, the jet's $p_{t}$ is modified, affecting the normalisation of $z$ in Eq. (11). Secondly, the heavy-ion background adds many extra particles to the jet, predominantly at low momenta.

\footnotetext{
${ }^{3}$ This tune is not the most up to date; however it is not unrealistic for the LHC and has the characteristic that it is based on Pythia's virtuality ordered shower, which is a prerequisite for use with Pyquen.

${ }^{4}$ In their notes, CMS and ATLAS often call this procedure 'embedding' or 'overlying' respectively.
} 
To appreciate the impact of the extra particles in the jet from the heavy-ion background, it is instructive to first examine the "Pythia+Hydjet" dashed red curves of Fig. 3, These show the FF extracted in heavy-ion collisions, without any subtraction of the background contribution to the $\mathrm{FF}$, but always using a $z$ value defined such that the jet's $p_{t}$ has been corrected for the expected HI background contamination (as is standard in the experimental measurements). At this stage we will not perform any unfolding to account for fluctuations in the HI background.

One sees how the FF acquires a 'bump' in the soft region, which lies at larger $\xi$ (smaller $z$ ) than the maximum in the original $p p$ result (blue solid line) and is up to two orders of magnitude higher. For the moments, this presence of the background is seen as a steep increase in the small- $N$ region, taking the curve far off the scale.

At large $z$ (small $\xi$ ), the impact of the addition of the background is only barely visible, as might be expected given that it is dominated by soft particles. However, this visually small effect is partially an artefact of the logarithmic scale used to show the FF versus $z$. Considering instead the moments, one sees that there is a non-negligible reduction in the $\mathrm{FF}$ at large $N$. This is perhaps surprising given that the background adds particles. It is a feature related to the interplay between background fluctuations and the steeply falling jet spectrum. It is well known by the experiments and we will return to discuss it in section 5 .

\section{$4.1 \quad z$-space subtraction}

The traditional approach to background subtraction from a jet FF involves the construction of a distribution (in $z$ or $\xi$ space) intended to approximate that of background-only particles, and the subtraction of this distribution from the measured one. The way the background-only distribution is determined can vary, the simplest one probably being to consider a region of the event that is expected to be little affected by the hard jets, and measure it there.

To illustrate $z$-space subtraction here, we measure the distribution of hadrons in two regions transverse in azimuth with respect to the axis defined by the dijet event. Event by event, and jet by jet, we subtract those distributions (measured in a patch of phase space with an area equal to that of the jet that one is considering) from the jet fragmentation function. While exact experimental procedures differ in the details, most choices lead to similar results here. Perhaps the main distinction of the experimental procedures is that they sometimes address issues related to flow, which for simplicity we neglect here in our $z$-space subtraction.

The results of this subtraction are shown in figure 3 as green open circles, labelled ' $z$-subtracted' (the moments of these $z$-subtracted results are also shown). One sees how these curves come closer to the $p p$ results at small $z$ and small $N$ than do the unsubtracted (dashed red) ones. The agreement improves as the jet $p_{t}$ threshold is increased. However, even with jets of $p_{t} \gtrsim 200 \mathrm{GeV}$, this procedure still falls short of an accurate reconstruction of the hard FF. In fact, the region in $z$ where the subtracted FF works well barely extends beyond that selected by simply truncating the unsubtracted FF at low $p_{t, \mathrm{~h}}$ so as to avoid the region dominated by the background.

\section{$4.2 \quad N$-space subtraction}

An alternative approach is to directly subtract the moments $M_{N}$ of the fragmentation function. This can be done by extending the jet-area based techniques for background estimation introduced in [38] and implemented in [36].

Let us first recall the procedure for subtracting the background contribution to a jet's $p_{t}$. One first determines the background $p_{t}$ density per unit area, $\rho$, for the event (or just part of the event 

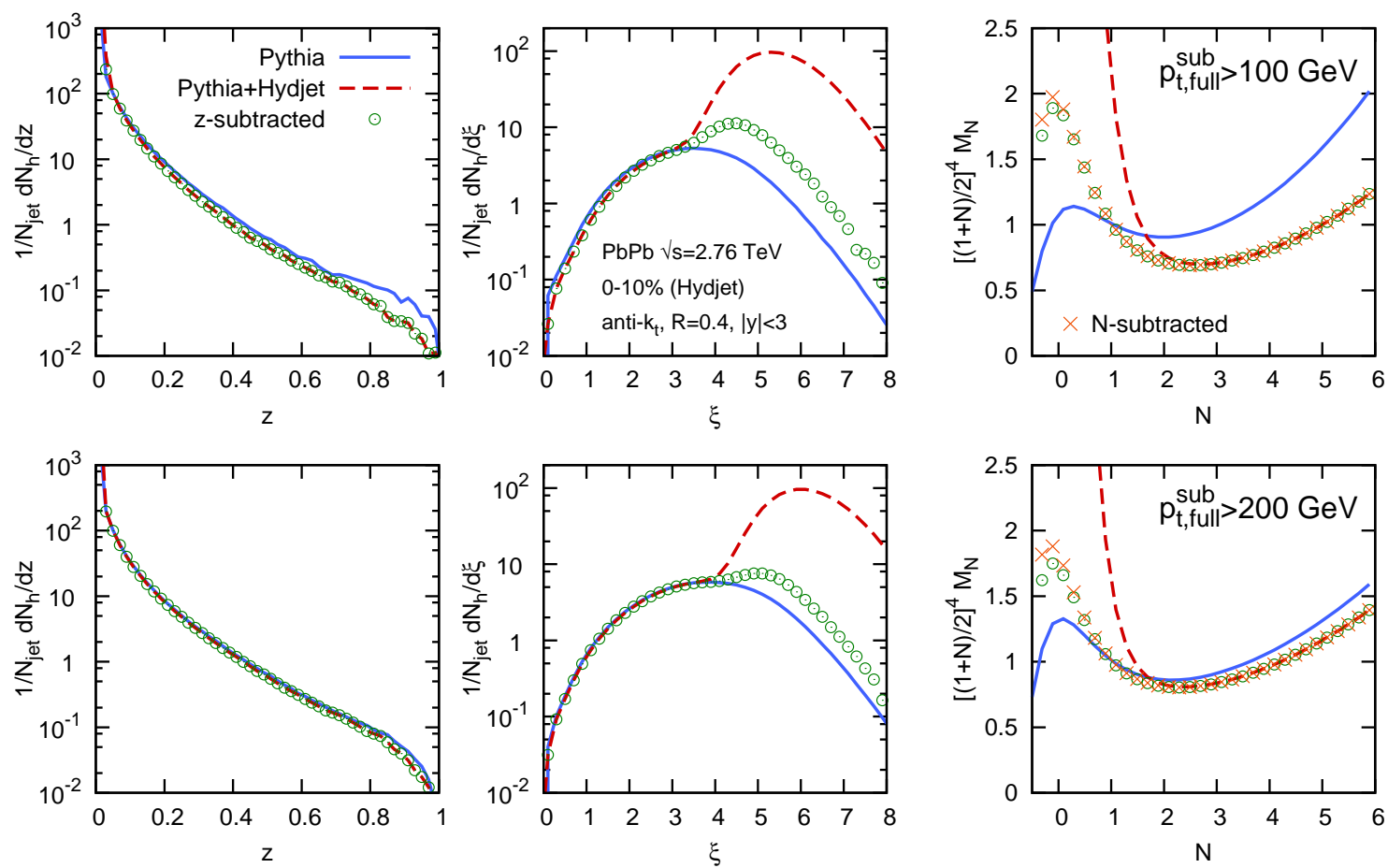

Figure 3: Jet fragmentation functions shown for plain Pythia, with the addition of the heavy-ion background (Pythia+Hydjet) and after subtraction of the heavy-ion background ( $z$-subtracted and $N$-subtracted). For the results including the heavy-ion background, the jet $p_{t}$ used to define $z$ is always that after subtraction of the heavy-ion background. As in Fig. 1, we show the results as a function of $z$ (left), $\xi$ (middle) and for the moments versus $N$ (right). The upper (lower) row has a jet $p_{t}$ threshold of $100 \mathrm{GeV}(200 \mathrm{GeV})$.

in the vicinity of the jet). One accomplishes this by dividing the event into patches of similar rapidity-azimuth area, e.g. by running the inclusive $k_{t}$ algorithm and taking all the jets it finds. Then $\rho$ is obtained by taking the median across all patches of the ratio of $p_{t}$ to area $\left(A_{\mathrm{patch}}\right)$ for each patch:

$$
\rho=\underset{\text { patches }}{\operatorname{median}}\left\{\frac{p_{t, \text { patch }}}{A_{\text {patch }}}\right\} .
$$

The median serves to limit biases from any hard jets among the patches. A given jet's full transverse momentum, $p_{t, \text { full }}$, which includes genuine jet particles and background particles, is then corrected for the background particles by subtracting an amount given by the product of $\rho$ and the jet's area A:

$$
p_{t, \text { full }}^{\text {sub }}=p_{t, \text { full }}-\rho A .
$$

We use patches in an annulus (or 'doughnut') of outer radius $3 R$ and inner radius $R$, centred on the jet of interest. This choice helps to limit corrections due to flow and accounts for the rapidity dependence of the background 5

For the purpose of correcting FF moments, the procedure we propose is quite similar. One first determines the expected background contribution per unit area to a given moment (or rather to its

\footnotetext{
${ }^{5}$ Alternatively one could determine a global $\rho$ for the event and modulate it for rapidity and azimuthal dependence, as discussed in [36], and similarly to what is done for example by ATLAS [42].
} 
numerator in Eq. (3) ):

$$
\rho_{N}=\underset{\text { patches }}{\operatorname{median}}\left\{\frac{\sum_{i \in \text { patch }} p_{t, i}^{N}}{A_{\text {patch }}}\right\} .
$$

For example, for $N=0$ this will be the median particle multiplicity per unit area. The subtracted FF moment is then obtained by separately taking the numerator and denominator of Eq. (3), as measured in the full event, and respectively subtracting $\rho_{N} A$ and $\rho A$ :

$$
M_{N}^{\mathrm{sub}}=\frac{\sum_{i \in \mathrm{jet}} p_{t, i}^{N}-\rho_{N} A}{\left(p_{t, \mathrm{full}}-\rho A\right)^{N}} .
$$

The results for $M_{N}^{\text {sub }}$ are shown in figure 3 in the right-most plots, orange crosses labelled ' $N$ subtracted'. One can see that they are neither better nor worse than the corresponding $z$-subtracted ones, which have been translated to $N$-space and drawn in the same plots for direct comparison (green circles).

In order to provide a fragmentation function reconstruction that is better than that given by the standard $z$-space method, we introduce in the next section an improved background subtraction method that is most straightforwardly applied in moment space.

\section{Improved background-subtracted fragmentation function}

The discrepancies that we have observed can, we believe, largely be attributed to fluctuations of the background.

Background fluctuations mean that neither the jet's $p_{t}$ nor the numerator of the FF moment are perfectly reconstructed for any given jet. When selecting jets above some $p_{t}$ threshold for a process with a steeply falling jet spectrum, it is favourable to select jets slightly below the $p_{t}$ threshold, but which have an upwards background fluctuation (cf. the discussion of section 5 of [40]). One consequence of this is that the denominator in Eq. (9) tends to be larger than the actual jet $p_{t}$. The larger the value of $N$, the greater the impact of this effect. One can also understand it as resulting in an underestimate of the $z$ fraction, leading to the FF being spuriously shifted to lower $z$. Such an effect is already well known: CMS [11, for example, when comparing with $p p$ FFs, explicitly applies a correction to the $p p$ FFs to account for the smearing of the denominator of the $z$ variable that is expected when the jet's momentum is reconstructed in a HI environment. ATLAS [28] carries out an unfolding to account for this.

A second consequence of fluctuations affects mostly the low- $z$, or low- $N$ region of the FF: the upwards fluctuations of the background $p_{t}$ that cause a jet to pass the $p_{t}$ cut even when it is below threshold also tend to be associated with upwards fluctuations of the multiplicity of soft background particles. It is this effect that causes the FF to be overestimated for low values of $N 6$

One way of verifying the above interpretation is to consider $\gamma+$ jet events, selecting events based on the photon $p_{t}$ and normalising the fragmentation function $z$ also to the photon $p_{t}$. In this case, the background-induced fluctuations of the jet's reconstructed $p_{t}$ are of no relevance, and in explicit simulations, we have found that plain subtraction is already quite effective.

For the dijet case, in the limit where the background fluctuations are reasonably small compared to the transverse momentum of the jet, it is possible to devise a simple correction in moment space

\footnotetext{
${ }^{6}$ As this letter was being finalised, ATLAS presented preliminary results [28] in which they correct for this by multiplying the expected background contribution by a $z$-independent factor that is a function of the jet $p_{t}$.
} 
for both kinds of fluctuation-induced bias. In order to do so, we start by rewriting the subtracted moments of the jet FF in Eq. (9) as

$$
M_{N}^{\mathrm{sub}}=\frac{\sum_{i} p_{t, i}^{N}-\rho_{N} A}{\left(p_{t, \text { full }}-\rho A\right)^{N}} \equiv \frac{S_{N}}{S_{1}^{N}}
$$

where we have introduced the shorthands $S_{N}$ for the subtracted numerator and $S_{1}$ for $p_{t, \text { full }}^{\text {sub }}$, as defined in Eq. (7). Given our assumption that background fluctuations are moderate, we can locally approximate the hard jet cross-section by an exponential rather than by a power in order to facilitate our analytical working,

$$
H\left(p_{t}\right) \equiv \frac{d \sigma}{d p_{t}}=\frac{\sigma_{0}}{\mu} \exp \left(-p_{t} / \mu\right)
$$

Next we take a Gaussian approximation for the spectrum of background transverse-momentum fluctuations from one jet to the next. Denoting the fluctuation by $q_{t}$, the distribution of $q_{t}$ is then

$$
B\left(q_{t}\right) \equiv \frac{d P}{d q_{t}}=\frac{1}{\sqrt{2 \pi A} \sigma} \exp \left(-\frac{q_{t}^{2}}{2 \sigma^{2} A}\right)
$$

where $\sigma$ is a parameter that describes the size of fluctuations from one patch of area 1 to another. It can be extracted directly from the event in a manner similar to $\rho$ [38, 43]. Its value is of the order of $\sigma \simeq 18 \mathrm{GeV}$ for our $\mathrm{PbPb} \mathrm{LHC}\left(\sqrt{S_{N N}}=2.76 \mathrm{TeV}\right)$ simulations (the same simulation leads to fluctuations of $\sigma_{\text {jet }} \simeq 11 \mathrm{GeV}$ for anti- $k_{t} R=0.4$ charged-track jets, in good agreement with the measurement from ALICE [44]).

We further introduce the variable $Q_{N}$ to denote the difference between the actual background contribution to $S_{N}$ in a specific jet and the expected contribution, $\rho_{N} A$, i.e.

$$
Q_{N}=\left(\sum_{i \in \text { jet (bkgd) }} k_{t, i}^{N}\right)-\rho_{N} A,
$$

where the sum runs just over the background constituents $k_{t, i}$ of the jet. By construction, $Q_{1}=q_{t}$. In practice $Q_{N}$ cannot be determined for a single jet, since we don't know which particles are the background ones, but its statistical properties can be determined by looking at many jets. The fluctuations of $Q_{N}$ are not independent of the fluctuations $q_{t}$ of the background's transverse momentum: there is a correlation coefficient $r_{N}$ between them, defined as

$$
r_{N}=\frac{\operatorname{Cov}\left(q_{t}, Q_{N}\right)}{\sqrt{\operatorname{Var}\left(q_{t}\right) \operatorname{Var}\left(Q_{N}\right)}},
$$

where $\operatorname{Var}(X)$ is the variance of the variable $X$ and $\operatorname{Cov}(X, Y)$ the covariance of $X$ and $Y$. Using the fact that $\operatorname{Var}\left(q_{t}\right)=\sigma^{2} A, \operatorname{Var}\left(Q_{N}\right)=\sigma_{N}^{2} A$, we have that the average value for $Q_{N}$ as a function of $q_{t}$ is

$$
\left\langle Q_{N}\right\rangle\left(q_{t}\right)=\frac{\operatorname{Cov}\left(q_{t}, Q_{N}\right)}{\operatorname{Var}\left(q_{t}\right)} q_{t}=r_{N} \frac{\sigma_{N}}{\sigma} q_{t}
$$

With these ingredients we can now correct for the fluctuation effects as follows. Firstly, we note that the cross section for a given reconstructed $S_{1}$ (i.e. subtracted jet $p_{t}$ ) is

$$
\frac{d \sigma}{d S_{1}}=\int d q_{t} H\left(S_{1}-q_{t}\right) B\left(q_{t}\right)
$$


where the integral allows us to deduce the probability distribution for the actual fluctuations $q_{t}$ given $S_{1}$ :

$$
\left.\frac{d P}{d q_{t}}\right|_{S_{1}}=\frac{H\left(S_{1}-q_{t}\right) B\left(q_{t}\right)}{\int d q_{t}^{\prime} H\left(S_{1}-q_{t}^{\prime}\right) B\left(q_{t}^{\prime}\right)}
$$

corresponding, with our approximations for $H\left(p_{t}\right)$ and $B\left(q_{t}\right)$ in Eqs. (11) and (12) respectively, to an average $q_{t}$ of

$$
\left\langle q_{t}\right\rangle=\frac{\sigma^{2} A}{\mu} .
$$

If we measure a certain value $S_{N}$ in a jet, then as a function of the $q_{t}$ fluctuation, the expected true hard contribution to it is

$$
S_{N}^{\mathrm{hard}}=S_{N}-\left\langle Q_{N}\right\rangle\left(q_{t}\right)=S_{N}-r_{N} \frac{\sigma_{N}}{\sigma} q_{t}
$$

where we have averaged over possible $Q_{N}$ values, given the $q_{t}$ fluctuation. To obtain our estimate for $M_{N}^{\text {hard }}$, this should be normalised by the $N^{\text {th }}$ power of the true hard $p_{t}$ of the jet, $\left(S_{1}-q_{t}\right)^{N}$ :

$$
M_{N}^{\mathrm{hard}}=\frac{S_{N}^{\mathrm{hard}}}{\left(S_{1}-q_{t}\right)^{N}}=\frac{S_{N}}{S_{1}^{N}}+N \frac{S_{N} q_{t}}{S_{1}^{N+1}}-r_{N} \frac{\sigma_{N} q_{t}}{\sigma S_{1}^{N}}+\mathcal{O}\left(q_{t}^{2}\right)
$$

One subtlety here is that this is an estimate for $M_{N}^{\text {hard }}$ in hard jets with true $p_{t}=S_{1}-q_{t}$. However because $M_{N}$ is a slowly varying function of $p_{t}$, by taking the result as contributing to $M_{N}^{\text {hard }}\left(S_{1}\right)$ rather than $M_{N}^{\text {hard }}\left(S_{1}-q_{t}\right)$ we make only a small mistake, of the same order as other terms that we shall neglect 7

Retaining the terms linear in $q_{t}$ in Eq. (20) and averaging now over possible $q_{t}$ values, making use of Eq. (18), leads to the following prescription for an "improved" subtracted $M_{N}\left(S_{1}\right)$, corrected for fluctuations effects up to first order in $q_{t} / S_{1}$ :

$$
M_{N}^{\mathrm{sub}, \mathrm{imp}}=M_{N}^{\mathrm{sub}} \times\left(1+N \frac{\sigma^{2} A}{S_{1} \mu}\right)-r_{N} \frac{\sigma \sigma_{N} A}{\mu S_{1}^{N}} .
$$

This is simpler than the corresponding correction would be directly in $z$ space, in particular because in $z$ space the correction to one bin of the fragmentation function depends in a non-trivial way on the contents of nearby bins. One can think of the advantages of moment space as being that the correction to a given $N$ value does not depend on $M_{N}$ at all other values of $N$, and that it is straightforward to account for correlations between fluctuations in the jet- $p_{t}$ and in the moments.

Note that in a real experimental context, calorimeter fluctuations of the reconstructed jet and background $p_{t}$ 's would have an effect akin to increasing $\sigma$ and decreasing the correlation coefficient $r_{N}$. The in-situ methods that we use for the determination of $\sigma, \sigma_{N}$ and $r_{N}$ would automatically take this into account. Noise-reduction methods in the reconstruction of the jet $p_{t}$, as used by CMS 2], would have the effect of reducing $\sigma$ (and probably also $r_{N}$ ). However noise reduction is

\footnotetext{
${ }^{7}$ This can be seen by observing that $M_{N}$ satisfies a DGLAP-style equation for its evolution $d M_{N} / d \ln p_{t} \sim \alpha_{s} M_{N}$. Given that $q_{t}$ is itself small compared to $p_{t}, M_{N}$ for jets with $p_{t}=S_{1}-q_{t}$ differs from that for jets with $p_{t}=S_{1}$ by a relative amount $\sim \alpha_{s} q_{t} / S_{1}$, which we can neglect in the same way that we neglect $\mathcal{O}\left(q_{t}^{2}\right)$ terms. If we wanted to improve on this approximation, then one approach might be, for each event, to use the middle expression in Eq. (20) and assign it to $M_{N}$ at $p_{t}=S_{1}-\left\langle q_{t}\right\rangle$. We have not, however, investigated this option in detail and other improvements would probably also be necessary at a similar accuracy, e.g. taking into account deviations from the simple exponential and Gaussian approximations that we have used for $H\left(p_{t}\right)$ and $B\left(q_{t}\right)$.
} 

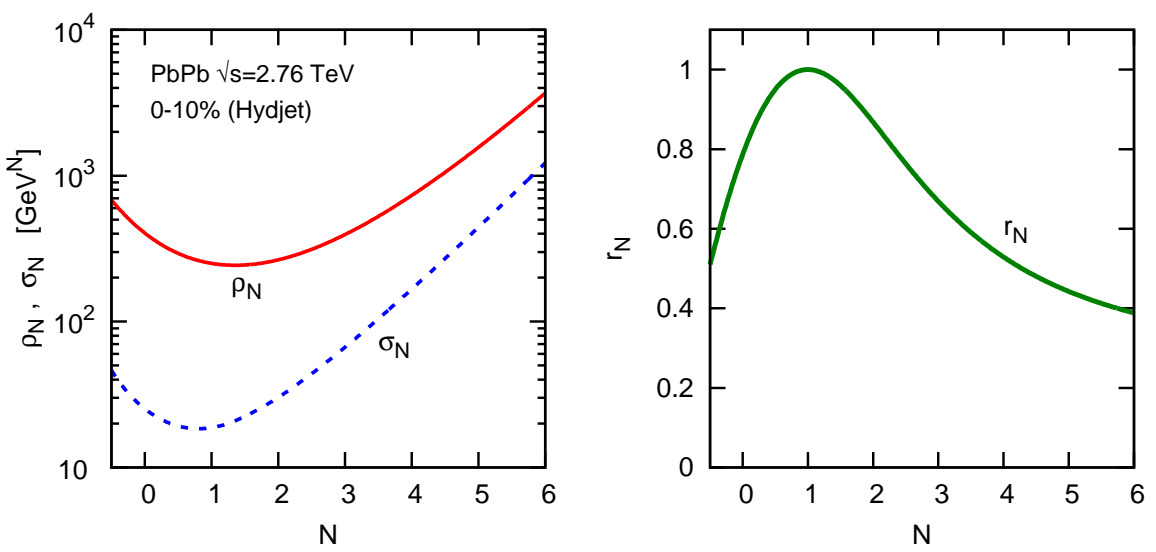

Figure 4: The quantities $\rho_{N}, \sigma_{N}$ and the correlation coefficient $r_{N}$, shown as a function of $N$ for $0-10 \%$ central $\mathrm{Pb} \mathrm{Pb}$ collisions $\sqrt{s_{N N}}=2.76 \mathrm{TeV}$ as obtained from simulations with Hydjet.

likely to complicate the meaningful determination of $r_{N}$, since it acts differently on pure background jets as compared to jets with a hard fragmenting component.

The correction in Eq. (21) can be applied jet-by-jet to correct for the fluctuation effects. It requires the prior knowledge of the slope $\mu$ of the jet cross-section, which can be obtained from $p p$ data, or from simulations 8 All the other ingredients that enter this equation $\left(\sigma, A, S_{N}, \sigma_{N}, r_{N}\right)$ can instead be determined event-by event or jet-by-jet. In practice we determine $\sigma, \sigma_{N}$ and $r_{N}$ from the ensemble of jets contained in an annulus (or "doughnut") of outer radius $3 R$ and inner radius $R$, centred on the jet of interest. Typical values of $\rho_{N}, \sigma_{N}$ and $r_{N}$ are presented as a function of $N$ in Fig. 4.

We show in figure 5 the result of applying Eq. (21) to our subtraction in moment space, for two jet $p_{t}$ thresholds. The solid blue curve ( $p p$ reference) and orange crosses (N-subtracted) are identical to the results in the rightmost plots in figure 3. In addition, figure 5 also displays results obtained using Eq. (21), shown as red diamonds. One sees how the quality of the agreement with the 'hard' blue curve is markedly improved. At low $N$ it is the last, additive, term in Eq. (21) that dominates this improvement, accounting for the correlation between a jet's reconstructed $p_{t}$ fluctuations and the fluctuations in the background's contribution to the moment; at high $N$ it is the multiplicative $N \sigma^{2} A / S_{1} \mu$ term that dominates, correcting for the fact that the more common upwards fluctuations in the jet's reconstructed $p_{t}$ cause the fragmentation $z$ value to be underestimated.

Figure 5 also shows (green dashed curve) the jet fragmentation function predicted by the quenching model used in Pyquen. One observes that the remaining deficiencies of the reconstruction are significantly smaller than the difference between the unquenched (solid blue) and the quenched (dashed green) FFs, pointing to a potential discriminating power. This is to be contrasted with subtraction without improvement (orange crosses), which especially in the soft region, $N<1$, fails to describe the blue curve sufficiently well to tell whether quenching (as predicted by Pyquen) is present or simply that an imperfect reconstruction is taking place. This serves as an illustration that the improved subtraction may now be sufficiently good to allow one to discriminate a quenched FF from an unquenched one.

We conclude this section by noting that an implementation of the tools needed to implement the

\footnotetext{
${ }^{8}$ In practice, $\mu$ depends on $p_{t}$ and should be taken at the scale $S_{1}-q_{t}$ in the integrand. However, $\mu$ varies slowly with $p_{t}$ and can easily be taken at the fixed scale $p_{t}$ in our small-fluctuations limit. In our analysis $\mu$ ranged from $\sim 9 \mathrm{GeV}$ at $p_{t} \simeq 50 \mathrm{GeV}$ to $\sim 28 \mathrm{GeV}$ at $p_{t} \simeq 200 \mathrm{GeV}$. At $p_{t} \simeq 100 \mathrm{GeV}$ we had $\mu \sim 16 \mathrm{GeV}$
} 

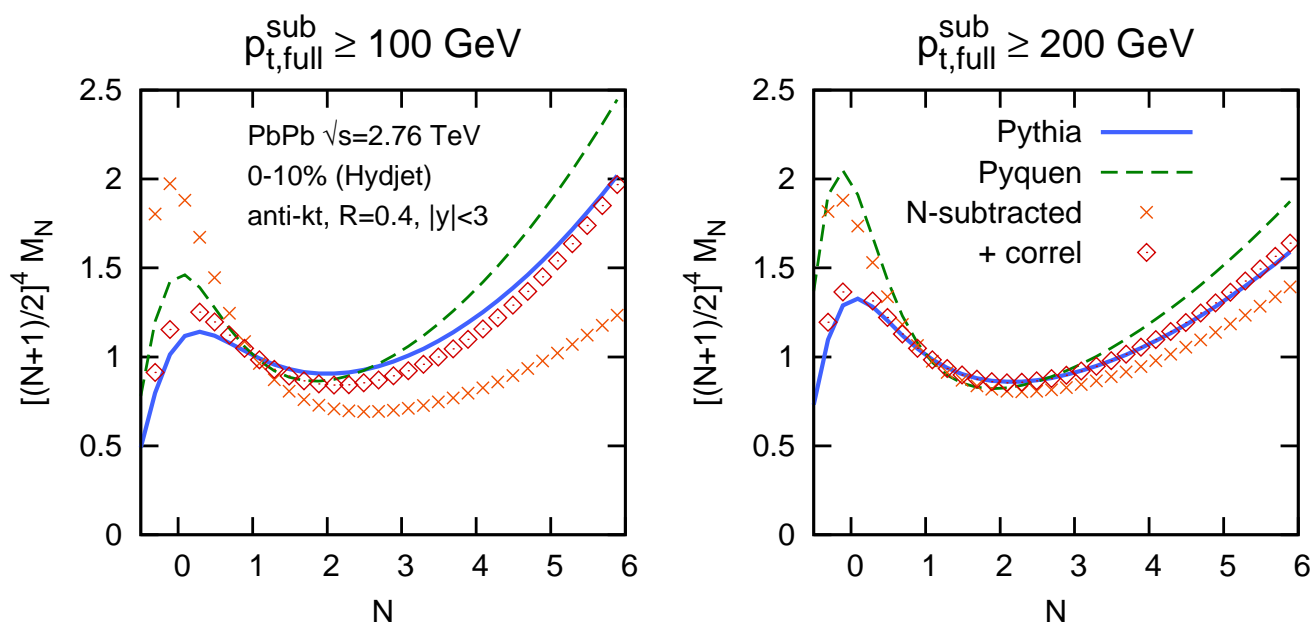

Figure 5: Jet fragmentation function moments, showing the plain Pythia result, the result after embedding in Hydjet and applying plain subtraction moment-space subtraction ("N-subtracted") and after the additional improvement to account for correlations ("+ correl"), Eq. (21). A quenched result ("Pyquen") is also shown, to help give an indication of the order of magnitude of quenching effects as compared to residual misreconstruction effects.

jet fragmentation function subtraction in moments space, as well as the fluctuations unfolding improvement, will be made available as a FastJet add-on from http://fastjet.hepforge.org/contrib.

\section{Conclusions}

In this paper we have suggested that it could be of interest to study jet fragmentation-function moments in heavy-ion collisions. Individual moments contain specific information such as jethadron multiplicities, the momentum fraction carried by charged particle and can also provide insight into the relation between inclusive hadron and inclusive spectra.

As we have seen, it is quite straightforward to correct moments for effects of fluctuating backgrounds, arguably more so than for fragmentation functions expressed in terms of momentum fractions. The correction procedure we discussed is amenable to systematic improvement, for example by accounting more completely for the shape of the jet $p_{t}$ spectrum. Such extensions are best considered as part of a more detailed analysis, for example in the context of a full experimental study.

\section{Acknowledgements}

This work was in part supported by the French Agence Nationale de la Recherche, under grants ANR-09-BLAN-0060 and ANR-10-CEXC-009-01 and by the EU ITN grant LHCPhenoNet, PITNGA-2010-264564. GPS wishes to thank Guilherme Milhano for related discussions. MC wishes to thank Peter Jacobs for useful conversations and comments on the draft. 


\section{References}

[1] G. Aad et al. [Atlas Collaboration], Phys. Rev. Lett. 105 (2010) 252303 arXiv:1011.6182 $[$ hep-ex]].

[2] S. Chatrchyan et al. [CMS Collaboration], Phys. Rev. C 84 (2011) 024906 arXiv:1102.1957 [nucl-ex]].

[3] S. Chatrchyan et al. [CMS Collaboration], Phys. Lett. B 712 (2012) 176 arXiv:1202.5022 [nucl-ex]].

[4] M. Cacciari, G. P. Salam and G. Soyez, Eur. Phys. J. C 71 (2011) 1692 arXiv:1101.2878 [hep-ph]].

[5] D. Buskulic et al. [ALEPH Collaboration], Phys. Lett. B 357 (1995) 487 [Erratum-ibid. B 364 (1995) 247].

[6] P. Abreu et al. [DELPHI Collaboration], Eur. Phys. J. C 6 (1999) 19.

[7] G. Abbiendi et al. [OPAL Collaboration], Eur. Phys. J. C 37 (2004) 25 hep-ex/0404026.

[8] F. Abe et al. Phys. Rev. Lett. 65, 968 (1990).

[9] D. Acosta et al. [CDF Collaboration], Phys. Rev. D 68 (2003) 012003.

[10] G. Aad et al. [ATLAS Collaboration], Eur. Phys. J. C 71, 1795 (2011) arXiv:1109.5816 [hep$\mathrm{ex}]]$.

[11] S. Chatrchyan et al. [CMS Collaboration], arXiv:1205.5872 [nucl-ex].

[12] N. Borghini and U. A. Wiedemann, hep-ph/0506218.

[13] X.-F. Guo and X.-N. Wang, Phys. Rev. Lett. 85, 3591 (2000) hep-ph/0005044.

[14] N. Armesto, L. Cunqueiro, C. A. Salgado and W. -C. Xiang, JHEP 0802, 048 (2008) arXiv:0710.3073 [hep-ph]].

[15] F. Arleo, Eur. Phys. J. C 61, 603 (2009) [arXiv:0810.1193 [hep-ph]].

[16] S. Sapeta and U. A. Wiedemann, Eur. Phys. J. C 55, 293 (2008) arXiv:0707.3494 [hep-ph]].

[17] A. Majumder, arXiv:0901.4516 [nucl-th].

[18] A. Beraudo, J. G. Milhano and U. A. Wiedemann, Phys. Rev. C 85 (2012) 031901 arXiv:1109.5025 [hep-ph]]; A. Beraudo, J. G. Milhano and U. A. Wiedemann, JHEP 1207 (2012) 144 [arXiv:1204.4342 [hep-ph]].

[19] I. P. Lokhtin and A. M. Snigirev, Eur. Phys. J. C 45 (2006) 211 arXiv:hep-ph/0506189.

[20] K. Zapp, G. Ingelman, J. Rathsman, J. Stachel and U. A. Wiedemann, Eur. Phys. J. C 60 (2009) 617 arXiv:0804.3568 [hep-ph]].

[21] T. Renk, Phys. Rev. C 78 (2008) 034908 arXiv:0806.0305 [hep-ph]]. T. Renk, Phys. Rev. C 79 (2009) 054906 arXiv:0901.2818 [hep-ph]]. 
[22] N. Armesto, L. Cunqueiro and C. A. Salgado, Eur. Phys. J. C 63 (2009) 679 arXiv:0907.1014 [hep-ph]].

[23] B. Schenke, C. Gale and S. Jeon, Phys. Rev. C 80 (2009) 054913 [arXiv:0909.2037 [hep-ph]].

[24] J. Putschke [STAR Collaboration], Eur. Phys. J. C 61, 629 (2009) [arXiv:0809.1419 [nucl-ex]].

[25] E. Bruna [STAR Collaboration], Nucl. Phys. A 830, 267C (2009) [arXiv:0907.4788 [nucl-ex]].

[26] CMS Collaboration, CMS-PAS-HIN-12-013.

[27] E. Etzion, on behalf of the ATLAS Collaboration, talk at ICHEP 2012, https://indico.cern.ch/contributionDisplay.py?contribId=210\&conf Id=181298

[28] Atlas Collaboration, ATLAS-CONF-2012-115.

[29] M. Cacciari and P. Nason, Phys. Rev. Lett. 89 (2002) 122003 hep-ph/0204025].

[30] M. Cacciari, G. P. Salam and G. Soyez, JHEP 0804 (2008) 063 [arXiv:0802.1189].

[31] T. Sjostrand, S. Mrenna and P. Skands, JHEP05 (2006) 026 hep-ph/0603175.

[32] I. P. Lokhtin, A. V. Belyaev, L. V. Malinina, S. V. Petrushanko, E. P. Rogochaya and A. M. Snigirev, arXiv:1204.4820 [hep-ph].

[33] M. G. Albrow et al. [TeV4LHC QCD Working Group], arXiv:hep-ph/0610012.

[34] I. P. Lokhtin and A. M. Snigirev, hep-ph/0312204.

[35] I. P. Lokhtin, L. V. Malinina, S. V. Petrushanko, A. M. Snigirev, I. Arsene and K. Tywoniuk, Comput. Phys. Commun. 180 (2009) 779 [arXiv:0809.2708].

[36] M. Cacciari, G. P. Salam and G. Soyez, Eur. Phys. J. C 72 (2012) 1896 arXiv:1111.6097 [hep-ph]], http://www.fastjet.fr.

[37] M. Cacciari and G. P. Salam, Phys. Lett. B 641 (2006) 57 hep-ph/0512210.

[38] M. Cacciari and G. P. Salam, Phys. Lett. B 659 (2008) 119 [arXiv:0707.1378].

[39] M. Cacciari, G. P. Salam and G. Soyez, JHEP 0804 (2008) 005 [arXiv:0802.1188].

[40] M. Cacciari, J. Rojo, G. P. Salam and G. Soyez, Eur. Phys. J. C 71 (2011) 1539 arXiv:1010.1759 [hep-ph]].

[41] S. Catani, Y. L. Dokshitzer, M. H. Seymour, and B. R. Webber, Nucl. Phys. B406 (1993) 187; S. Catani, Y. L. Dokshitzer, M. Olsson, G. Turnock, and B. R. Webber, Phys. Lett. B269 (1991) 432; S. D. Ellis and D. E. Soper, Phys. Rev. D48 (1993) 3160-3166, hep-ph/9305266.

[42] G. Aad et al. [ATLAS Collaboration], arXiv:1208.1967 [hep-ex].

[43] M. Cacciari, G. P. Salam and S. Sapeta, JHEP 1004, 065 (2010) [arXiv:0912.4926.

[44] B. Abelev et al. [ALICE Collaboration], JHEP 1203 (2012) 053 [arXiv:1201.2423 [hep-ex]]. 